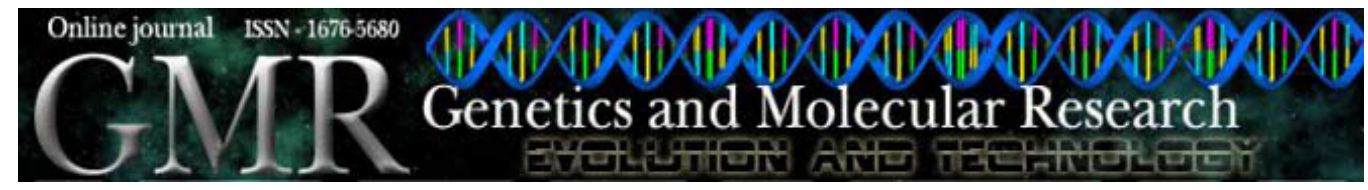

\title{
Mutation in intron 5 of GTP cyclohydrolase 1 gene causes dopa-responsive dystonia (Segawa syndrome) in a Brazilian family
}

\author{
C.P. Souza ${ }^{1}$, E.R. Valadares ${ }^{2}$, A.L.C. Trindade ${ }^{1}$, V.L. Rocha ${ }^{2}$, L.R. Oliveira ${ }^{3}$ \\ and A.L.B. Godard ${ }^{1}$ \\ ${ }^{1}$ Departamento de Biologia Geral, Laboratório de Genética Animal e Humana, \\ Instituto de Ciências Biológicas, Universidade Federal de Minas Gerais, \\ Belo Horizonte, MG, Brasil \\ ${ }^{2}$ Departamento de Propedêutica Complementar, Faculdade de Medicina, \\ Universidade Federal de Minas Gerais, Belo Horizonte, MG, Brasil \\ ${ }^{3}$ Departamento de Pediatria, Faculdade de Medicina, \\ Universidade Federal de Minas Gerais, Belo Horizonte, MG, Brasil \\ Corresponding author: A.L.B. Godard \\ E-mail: brunialti@icb.ufmg.br \\ Genet. Mol. Res. 7 (3): 687-694 (2008) \\ Received April 14, 2008 \\ Accepted June 23, 2008 \\ Published August 5, 2008
}

\begin{abstract}
Dopa-responsive dystonia (DRD), also known as Segawa syndrome or hereditary progressive dystonia with diurnal fluctuation, is clinically characterized by the occurrence of simultaneous or late Parkinsonism and by an excellent response to treatment with low doses of L-dopa. Diagnosis of DRD is essentially clinical. It is based on clinical history and the response to treatment with low doses of L-dopa. However, due to the low penetrance of the disease, asymptomatic carriers may exist. In these cases, mutational analysis of the $\mathrm{GCH} 1$ gene is an alternative to diagnose DRD. In the present study, we investigated a large DRD-carrier family in an attempt to identify the disease-causing mutation. The proband, a young woman diagnosed at the age of 13 years, is the daughter of a healthy non-consanguineous couple with history of several cases, on the maternal side of the family, of tip-toeing, disturbance of gait, Parkinsonism, rigidity and cramps in the lower limbs. Using single
\end{abstract}


strand conformational polymorphism and DNA sequencing techniques to analyze DNA extracted from blood samples, we identified a mutation in the GCH1 gene, IVS5+3insT, which would preclude the formation of the active enzyme due to the formation of truncated peptides.

Key words: Dopa-responsive dystonia; Segawa syndrome; GCH1 gene; GTP cyclohydrolase; Negative dominant effect; Hereditary progressive dystonia with diurnal fluctuation

\section{INTRODUCTION}

Dopa-responsive dystonia (DRD), also known as Segawa syndrome or hereditary progressive dystonia with diurnal fluctuation, was described for the first time by Segawa and colleagues in 1971. The disease is clinically characterized by the occurrence of simultaneous or late Parkinsonism and by an excellent response to treatment with low doses of L-dopa. In most cases, clinical manifestations start in childhood with dystonia, more frequently, in the lower limbs (Van Hove et al., 2006). In these cases, DRD phenotype may be similar to atypical cerebral palsy. Thus, about $24 \%$ of the patients with DRD are incorrectly diagnosed with cerebral palsy (Jan, 2004). In adults, Parkinsonism can be the main or the only symptom. Segawa syndrome is a rare disorder and occurs at a frequency of 1 in 2 million (Nygaard, 1993). However, this rate is probably underestimated due to, at least, two reasons: the first one is that the disease is not easily recognized by physicians; the second reason lays in the fact that many signs and symptoms are not severe enough for patients to seek clinical advice (Bianca and Bianca, 2006).

DRD can be inherited as an autosomal dominant or in a recessive manner, depending on the mutated gene (locus heterogeneity). The recessive condition is caused by a mutation in the tyrosine hydroxylase gene, which is a mono-oxygenase that catalyzes the conversion of L-tyrosine into L-dopa (De Lonlay et al., 2000). In this study we emphasized the dominant condition caused, mainly, by mutations in the guanosine triphosphate cyclohydrolase 1 gene (GCH1), located on chromosome 14q22.1-q22.2 (Nygaard et al., 1993). The disease, inherited as an autosomal dominant, exhibits reduced penetrance, highly variable expressivity and, it is estimated that women are two to four times more affected than men (Ichinose et al., 1994). The GCH1 gene is constituted by 6 exons; it occupies approximately $30 \mathrm{~kb}$ in the genomic DNA and, at least six differential splicing variants differing on the 3' end have been described (Togari et al., 1992; Golderer et al., 2001; Hwu et al., 2003). However, only the full length transcript exhibits enzymatic activity (Gütlich et al., 1994). This transcript codes GTP cyclohydrolase I, an enzyme involved in the regulation of tetrahydrobiopterin $\left(\mathrm{BH}_{4}\right)$ synthesis, an essential co-factor for hydroxylases that converts phenylalanine, tryptophan, and tyrosine into tyrosine, serotonin and L-dopa, respectively (Müller et al., 2002).

Diagnosis of DRD is essentially clinical, based on the clinical history of the patient and the response to treatment with low doses of L-dopa. However, due to the low penetrance of the disease, asymptomatic carriers may exist. In these cases, the mutational analysis of the $\mathrm{GCH} 1$ gene is an alternative to detect asymptomatic and oligosymptomatic carriers. Alterations in the gene sequence have been found, through single strand confor- 
mation polymorphism (SSCP) analyses, in about 50 to $60 \%$ of clinically identified cases (Hagenah et al., 2005). Recently, a heterozygous deletion, not detectable through conventional sequencing, was identified by Southern blot analysis (Furukawa et al., 2000).

The objective of this study was to identify the mutation which causes DRD in individuals of an affected family.

\section{MATERIAL AND METHODS}

\section{Patients}

Fifty-five individuals from the same DRD Brazilian family were studied. The proband was a young woman diagnosed when she was 13 years old, who was the daughter of a healthy non-consanguineous couple with history of several cases of tip-toeing, disturbance of gait, Parkinsonism, rigidity and cramps in the lower limbs on the maternal side of the family. It is known that her pregnancy and delivery were free of incidences. Her symptomatology started at around 1 year old with dystonia in the left foot, progressively evolving to dystonia of the lower limbs. The affected patient was submitted to a posterior left tibial tenotomy on the age of 10 years. At 13 years, she could not walk more than one block, and she complained about an almost constant lack of balance and decrease in spasticity when at rest. The patient had previously shown unaltered results in electromyography, brain tomography and encephalic magnetic resonance. She had been incorrectly diagnosed by several neurologists with spastic diplegia, and physical therapy was the only treatment indicated for her. In the objective examination, she showed dystonia of both lower limbs, mild axial dystonia, flatfeet, exaggerated tendon reflexes and Babinski sign. Total remission of symptoms was achieved when the treatment with L-dopa was initiated ( $30 \mathrm{mg}$ twice a day, orally). The diagnosis for DRD was then established. The same treatment was indicated for all affected and symptomatic relatives. Blood samples of all fifty-five individuals were collected for DNA extraction.

The participants were fully informed and participated voluntarily. The project was approved by the Research Ethics Committee of the Universidade Federal de Minas Gerais under the number CAAE - 0428.0.203.000-05, February 15, 2006.

\section{Molecular analyses}

Genomic DNA was extracted from $10 \mathrm{~mL}$ peripheral blood according to a standard protocol: lysis buffer (10 mM Tris-HCl, $\mathrm{pH} 8.0,2 \mathrm{mM}$ EDTA, $\mathrm{pH} 8.2$, and 10\% SDS) and proteinase $\mathrm{K}(20 \mathrm{mg} / \mathrm{mL})$. Then, the pellet was washed with $70 \%$ ethanol and dissolved in TE (Miller et al., 1988). The DNA was quantified by spectrophotometry at $260 \mathrm{nM}$.

The exons of the GCH1 gene, including the splicing sites, were amplified from genomic DNA using polymerase chain reaction with previously described primers (Ichinose et al., 1995). For identification of the mutated fragment, the SSCP analysis was applied to the amplification products of the family members and the individual control. The fragment that exhibited a different migration pattern in the gel when the two groups were analyzed was cloned into the $\mathrm{pCR}^{\circledR} \mathrm{II}-\mathrm{TOPO}{ }^{\circledR}$ plasmid using the TOPO TA Cloning Kit, as recommended by manufacturer instructions (Invitrogen ${ }^{\mathrm{TM}}$ Life Technologies, Carlsbad, CA, USA) and, then, both alleles were sequenced in order to identify the muta- 
tion causing the disease. Sequencing was done with the same primers used in the initial amplification, in a MegaBACE $1000^{\circledR}$ system (GE Healthcare) using the DYEnamic $\mathrm{ET}^{\circledR}$ Terminator kit according to manufacturer instructions. Both strands of the DNA samples were sequenced. The sequences obtained were first converted into the ab1file format, using the Chromas software (www.technelysium.com.au/chromas_lite.html), and then analyzed with the CodonCode Aligner program (www.codoncode.com/aligner). The fragment containing exon 1 was directly sequenced since, because of its size (502 bp), it was not adequate for the SSCP technique.

\section{RESULTS}

The characteristic of an autosomal dominant inheritance can be observed in Figure 1, which shows the pedigree of the family. Except for exon 5, the migration pattern of the fragments on the SSCP gel did not exhibit differences between the individuals studied and the control sample. Figure 2 shows that, individuals VI-3, IV-35, III-12, and V-16 presented the same migration pattern as the individual control, thus, they, probably, do not have a mutation. The remaining individuals showed an extra band, and are, therefore, carriers of the mutation.

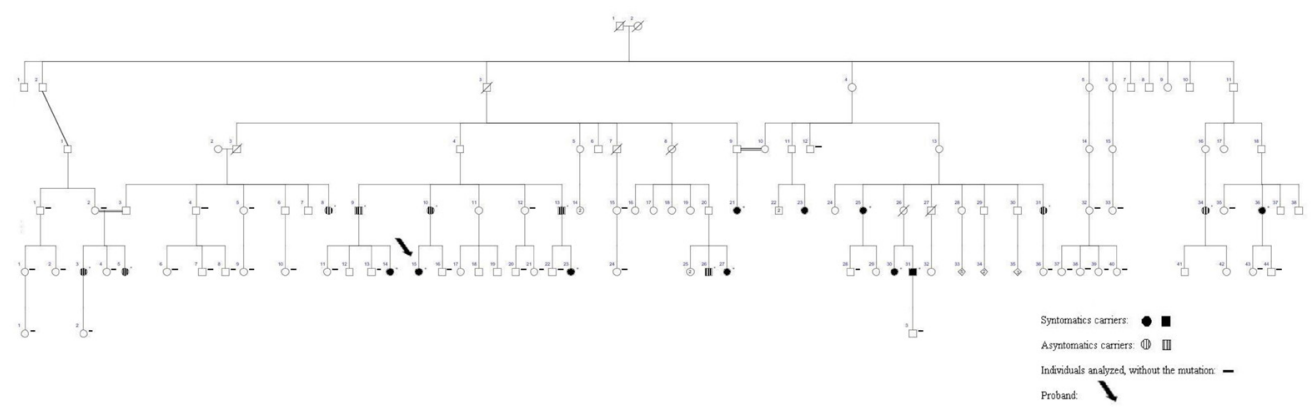

Figure 1. Pedigree of a Brazilian family with dopa-responsive dystonia. Nineteen of the fifty-five individuals analyzed are mutation carriers.

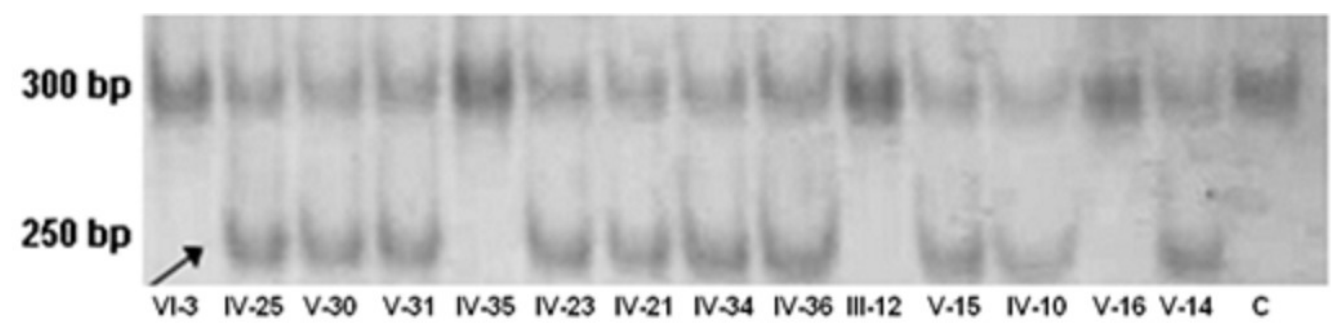

Figure 2. Single strand conformation polymorphism gel showing the fragment containing exon 5 of the GCH1 gene. The arrow indicates the band that differs from the banding pattern observed in the control sample (C). Individuals who have the mutation show two bands. The numeration is in accordance with the pedigree in Figure 1. 
Direct sequencing of the fragment containing exon 1 did not indicate any mutation in DNAs analyzed. On the other hand, sequencing of the fragment containing exon 5 showed a heterozygous splice site mutation, the insertion of a thymine next to the splice site of intron 5. Figure 3 shows the chromatogram with the sequencing of the cloned fragment of exon 5 from the individual with the mutation.

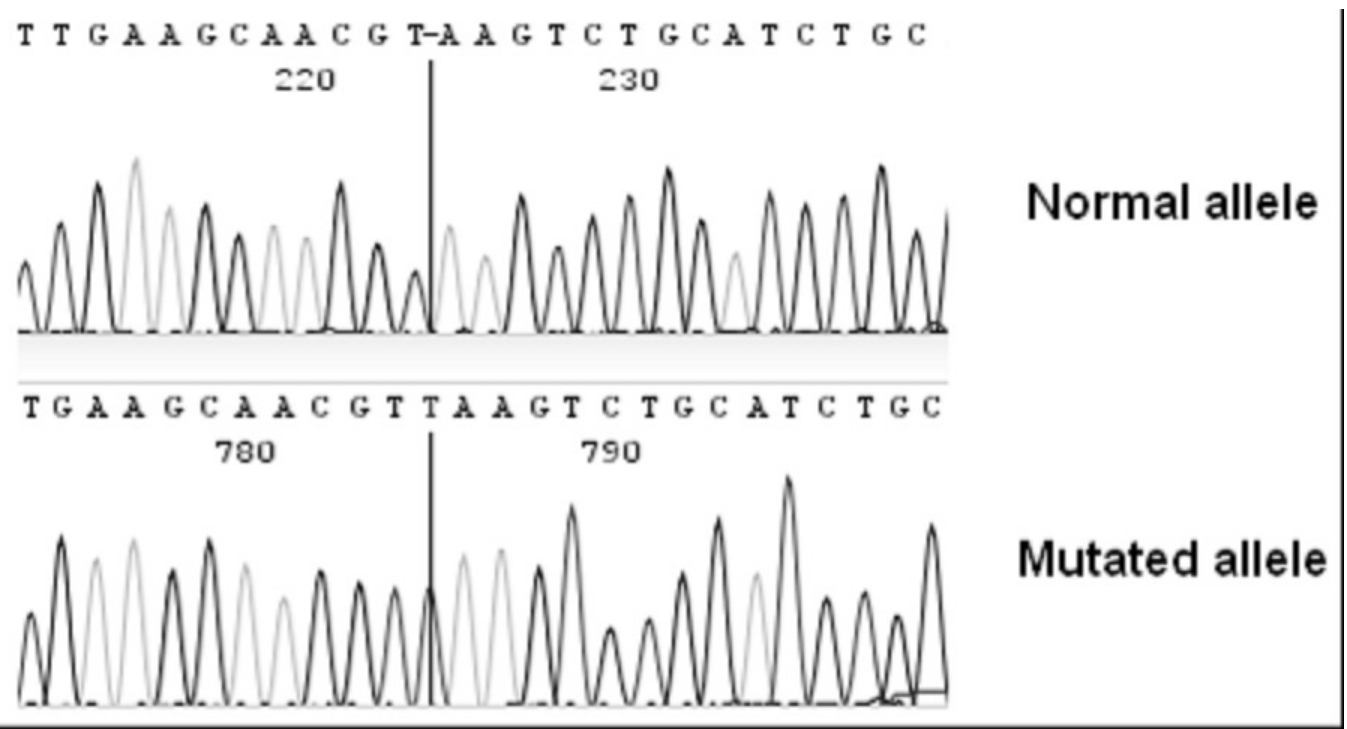

Figure 3. Chromatograms of the sequencing of the exon 5 of the GCH1 gene from a mutated individual that was cloned (IV-23). Comparing with the GCH1 dataset (www.ensembl.org), it is observed that the mutated allele shows one more peak that represents an insertion of a thymine.

The mutation IVS5+3insT in the GCH1 gene was identified in 19 individuals, 15 women and 4 men, of the 55 individuals studied. Nine women were symptomatic ( $60 \%$ of the detected women), and only 1 man ( $25 \%$ of the detected men).

\section{DISCUSSION}

More than 85 mutations in the GCH1 gene have been described so far (http://www. bh4.org/BH4_databases_biomdb2.asp) and most of them reside in the coding region of the gene. Abnormalities have been reported in all 6 exons of the gene and in the intron splicing sites (Nishiyama et al., 2000). In this study, the mutation in the intron 5 splicing site of the GCH1 gene, IVS5+3insT, was identified in 19 individuals of a Brazilian family, as the one causing DRD. This mutation has already been described in patients with DRD (Kaindl et al., 2005). The insertion probably precludes the intron 5 remotion and causes the introduction of a premature stop codon resulting in a truncated peptide without exon 6 .

The enzyme guanosine triphosphate cyclohydrolase 1 is a protein composed of ten identical 250 amino acid monomers, coded by the GCH1 gene. The homodecamer is formed 
by five dimers organized in a ring structure that looks like two pentamers aligned face to face (Nar et al., 1995). Figure 4 shows a diagram with the relationship between the exons of the GCH1 gene and the structural part of the peptide for which they code. The study of Swick and Kapatos, published in 2006, showed that the dimers associate in a decamer through the interaction between $\mathrm{b} 1$ of one of the monomers with $\mathrm{b} 4$ of another monomer from an adjacent dimer. In addition, they have also concluded from their experiments that h6 would also stabilize the decamer. Based on Figure 4 it is possible to infer that the truncated peptide would be deficient in $\mathrm{h} 6 \alpha$-helices and b3 and b4 $\beta$-sheets that correspond to exon 6 . This deficiency would prevent the formation of the decamer through the dimer interaction, since, as shown previously, this interaction takes place between segments b1 and b4 of the monomer. Biochemical analyses showed that only the GCH1 decamer exhibits enzymatic activity (Yim and Brown, 1976); this fact is explained by structural studies that indicate the existence of 10 active sites formed on the interface of three separate monomers, located in adjacent dimers (Nar et al., 1995). Thus, it can be suggested that the product coded by the mutated gene would not show enzymatic activity. Another fact that supports this hypothesis is that the truncated peptide would not code one of the amino acids, Cys 212, which is part of the active site (Auerbach et al., 2000).

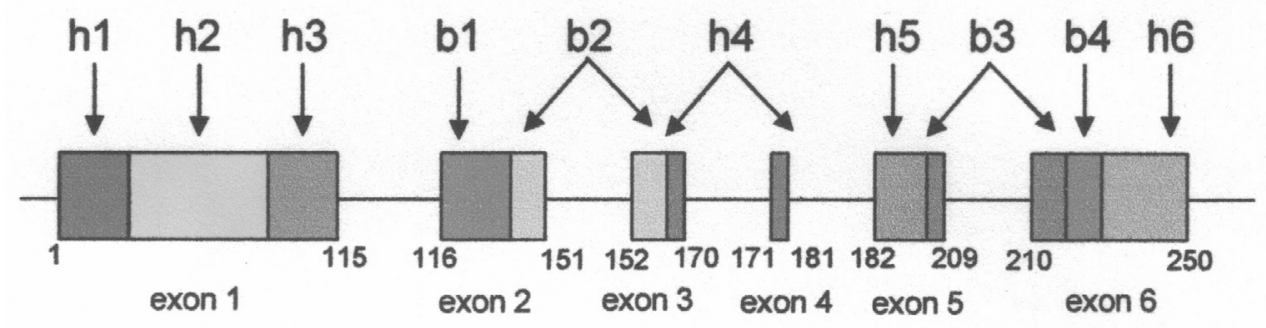

Figure 4. Diagram showing the correspondence between exons and structural parts of the GTPCH1 peptide. Each monomer folds into an N-terminal $\alpha$-helix (h1) and two anti-parallel helices $(\mathrm{h} 2, \mathrm{~h} 3)$, that are separated by a compressed C-terminal domain, composed of four anti-parallel $\beta$-sheets (b1-b4) divided by two anti-parallel helices (h4, h5) that end in a C-terminal helix (h6) (Nar et al., 1995).

Many inborn errors of the metabolism are recessive because half of the enzymatic activity is enough to produce or metabolize a certain substance (Suzuki et al., 1999). In DRD this haplosufficiency is not observed and it is estimated that the activity of GTP cyclohydrolase in the brain is reduced to $20 \%$ compared to normal individuals (Suzuki et al., 1999), generating a dominant inherited disease. One hypothesis that was believed initially to explain the observed fact was the possibility that the mutant and normal peptides interacted forming a non-functional heterodecamer, i.e., the mutant peptide would exert a negative dominant effect over the wild type (Hirano and Ueno, 1999). These authors were able to demonstrate this effect for some mutations in co-transfection experiments with wild-type and mutated GCH1 cDNAs. However, some mutations have been described, which code peptides apparently unable to interact with wild-type peptides (Suzuki et al., 1999). Experiments done by Hwu and colleagues, in 2003, 
have indicated that the presence of GCH1 transcript isoforms, type II mRNA - which does not show enzymatic activity - contributes to the regulation of the gene by reducing the basal activity of the protein through a dominant negative effect. Thus, another possible hypothesis would be that in DRD patients, because of the decrease in the amount of a functional protein (type I), there is an increase in the type II mRNA/type I mRNA ratio resulting in a greater chance of assembling non-functional heterodecamers (Hirano et al., 1997). Since the peptide coded by the mutated allele described here would, probably, be unable to form heterodecamers due to the reasons mentioned above, the second hypothesis would be more adequate to explain the haploinsufficiency of the normal allele in this case.

In the group of 19 individuals with the mutation, women were symptomatic in $60 \%$ of cases (9 in 15) and men in 25\% (1 in 4).

In summary, we have identified the mutation at the GCH1 gene, IVS5+3insT, as the cause of DRD in a Brazilian family.

\section{ACKNOWLEDGMENTS}

We thank all members of the studied family that willingly participated in this research. Research supported by Coordenação de Aperfeiçoamento de Pessoal de Nível Superior (CAPES) and Fundação de Amparo à Pesquisa do Estado de Minas Gerais (FAPEMIG), Brazil.

\section{REFERENCES}

Auerbach G, Herrmann A, Bracher A, Bader G, et al. (2000). Zinc plays a key role in human and bacterial GTP cyclohydrolase I. Proc. Natl. Acad. Sci. U. S. A. 97: 13567-13572.

Bianca S and Bianca M (2006). A new deletion in autosomal dominant guanosine triphosphate cyclohydrolase I deficiency gene - Segawa disease. J. Neural Transm. 113: 159-162.

De Lonlay P, Nassogne MC, van Gennip AH, van Cruchten AC, et al. (2000). Tyrosine hydroxylase deficiency unresponsive to L-dopa treatment with unusual clinical and biochemical presentation. J. Inherit. Metab. Dis. 23: 819-825.

Furukawa Y, Guttman M, Sparagana SP, Trugman JM, et al. (2000). Dopa-responsive dystonia due to a large deletion in the GTP cyclohydrolase I gene. Ann. Neurol. 47: 517-520.

Golderer G, Werner ER, Heufler C, Strohmaier W, et al. (2001). GTP cyclohydrolase I mRNA: novel splice variants in the slime mould Physarum polycephalum and in human monocytes (THP-1) indicate conservation of mRNA processing. Biochem. J. 355: 499-507.

Gütlich M, Jaeger E, Rucknagel KP, Werner T, et al. (1994). Human GTP cyclohydrolase I: only one out of three cDNA isoforms gives rise to the active enzyme. Biochem. J. 302 (Pt. 1): 215-221.

Hagenah J, Saunders-Pullman R, Hedrich K, Kabakci K, et al. (2005). High mutation rate in dopa-responsive dystonia: detection with comprehensive GCHI screening. Neurology 64: 908-911.

Hirano M and Ueno S (1999). Mutant GTP cyclohydrolase I in autosomal dominant dystonia and recessive hyperphenylalaninemia. Neurology 52: 182-184.

Hirano M, Imaiso Y and Ueno S (1997). Differential splicing of the GTP cyclohydrolase I RNA in dopa-responsive dystonia. Biochem. Biophys. Res. Commun. 234: 316-319.

Hwu WL, Yeh HY, Fang SW, Chiang HS, et al. (2003). Regulation of GTP cyclohydrolase I by alternative splicing in mononuclear cells. Biochem. Biophys. Res. Commun. 306: 937-942.

Ichinose H, Ohye T, Takahashi E, Seki N, et al. (1994). Hereditary progressive dystonia with marked diurnal fluctuation caused by mutations in the GTP cyclohydrolase I gene. Nat. Genet. 8: 236-242.

Ichinose H, Ohye T, Matsuda Y, Hori T, et al. (1995). Characterization of mouse and human GTP cyclohydrolase I genes. Mutations in patients with GTP cyclohydrolase I deficiency. J. Biol. Chem. 270: 10062-10071.

Jan MM (2004). Misdiagnoses in children with dopa-responsive dystonia. Pediatr. Neurol. 31: 298-303.

Kaindl AM, Steinberger D, Heubner G, Müller U, et al. (2005). Phenotype of five patients with dopa-responsive dystonia and mutations in GCH1. J. Pediatr. Neurol. 3: 83-87. 
Miller SA, Dykes DD and Polesky HF (1988). A simple salting out procedure for extracting DNA from human nucleated cells. Nucleic Acids Res. 16: 1215.

Müller U, Steinberger D and Topka H (2002). Mutations of GCH1 in Dopa-responsive dystonia. J. Neural Transm. 109: 321-328.

Nar H, Huber R, Meining W, Schmid C, et al. (1995). Atomic structure of GTP cyclohydrolase I. Structure 3: 459-466.

Nishiyama N, Yukishita S, Hagiwara H, Kakimoto S, et al. (2000). Gene mutation in hereditary progressive dystonia with marked diurnal fluctuation (HPD), strictly defined dopa-responsive dystonia. Brain Dev. 22 (Suppl. 1): S102-S106.

Nygaard TG (1993). Dopa-responsive dystonia. Delineation of the clinical syndrome and clues to pathogenesis. $A d v$. Neurol. 60: 577-585.

Nygaard TG, Wilhelmsen KC, Risch NJ, Brown DL, et al. (1993). Linkage mapping of dopa-responsive dystonia (DRD) to chromosome 14q. Nat. Genet. 5: 386-391.

Segawa M, Ohmi K, Itoh S, Aoyama M, et al. (1971). Childhood basal ganglia disease with remarkable response to L-Dopa, hereditary basal ganglia disease with marked diurnal fluctuation. Shinryo 24: 667-672.

Suzuki T, Ohye T, Inagaki H, Nagatsu T, et al. (1999). Characterization of wild-type and mutants of recombinant human GTP cyclohydrolase I: relationship to etiology of dopa-responsive dystonia. J. Neurochem. 73: 2510-2516.

Swick L and Kapatos G (2006). A yeast 2-hybrid analysis of human GTP cyclohydrolase I protein interactions. $J$. Neurochem. 97: 1447-1455.

Togari A, Ichinose H, Matsumoto S, Fujita K, et al. (1992). Multiple mRNA forms of human GTP cyclohydrolase I. Biochem. Biophys. Res. Commun. 187: 359-365.

Van Hove JL, Steyaert J, Matthijs G, Legius E, et al. (2006). Expanded motor and psychiatric phenotype in autosomal dominant Segawa syndrome due to GTP cyclohydrolase deficiency. J. Neurol. Neurosurg. Psychiatry 77: 18-23.

Yim JJ and Brown GM (1976). Characteristics of guanosine triphosphate cyclohydrolase I purified from Escherichia coli. J. Biol. Chem. 251: 5087-5094. 